\title{
Genetic Heterogeneity in Ductal Carcinoma of the Breast
}

\author{
Jack H. Lichy, Fabienne Dalbègue, Maryam Zavar, Constance Washington, \\ Mark M. Tsai, Zong-Mei Sheng, and Jeffery K. Taubenberger \\ Molecular Pathology Division, Department of Cellular Pathology, Armed Forces Institute of Pathology, \\ Washington, DC
}

SUMMARY: Genetic heterogeneity in breast cancer has been observed both by cytogenetic and loss of heterozygosity (LOH) analyses; however, the frequency with which genetically heterogeneous clones arise is unknown. In this study, a panel of 115 breast carcinomas was analyzed to determine the extent of clonal divergence in tumor foci at progressive stages of tumor evolution. Intraductal, infiltrating, and metastatic tumor components were microdissected from each tumor and tested for LOH at 20 microsatellite markers on seven chromosomal arms. Of these cases, 24 (21\%) demonstrated genetically divergent clones during tumor progression. Clonal divergence, inferred from discordant LOH patterns, was observed most commonly between intraductal and infiltrating tumor (18 cases), but was also demonstrated between infiltrating and metastatic tumor (11 cases). Discordant LOH was observed with markers on one chromosomal arm in 16 cases, on two in 7 cases, and on four in 1 case, and was observed most commonly with markers on 17p, 17q, and 16q. More detailed microdissection of four cases provided evidence for a specific chronology of genetic alterations occurring during the progression of each tumor. The results indicate that the different tumor components observed microscopically in breast cancer specimens often represent genetically divergent clones. (Lab Invest 2000, 80:291-301).

$E$ lucidation of the sequence of genetic events responsible for progression of breast cancer from in situ to infiltrating and metastatic carcinoma is an important goal of efforts to understand the biological basis of this common malignancy. Progression is believed to occur through the accumulation of genetic changes via a process of clonal evolution and clonal selection (Brenner and Aldaz, 1997; Nowell, 1976). Surgically resected breast carcinoma specimens provide a unique resource for analyzing the genetic changes that occur during progression, because specimens typically contain foci of tumor in various stages of progression, including in situ carcinoma, invasive tumor, and lymph node metastases. Morphologically normal epithelial constituents of the breast are usually represented in such specimens, and foci of benign proliferative epithelial lesions may also be present.

Detailed studies of colon cancer have shown that adenomatous epithelium adjacent to carcinoma typically has some but not all of the genetic lesions

\footnotetext{
Received September 22, 1999.

Supported by Grant \#DAMD 17-94-J-4330 from the U. S. Army Medical Research and Materiel Command and by the intramural funds of the AFIP. The opinions or assertions contained herein are the private views of the authors and are not to be construed as official or as reflecting the views of the Department of the Army or the Department of Defense. This is U.S. Government work; there are no restrictions on its use.

Address reprint requests to: Dr. J. H. Lichy, Molecular Pathology Division, Department of Cellular Pathology, Armed Forces Institute of Pathology, 14th Street and Alaska Avenue, NW, Washington, DC 20306-6000. Fax: 202-782-7623; E-mail: lichy@afip.osd.mil
}

present in the fully developed malignancy, consistent with direct progression from adenoma to carcinoma (Boland et al, 1995; Fearon and Vogelstein, 1990; Vogelstein et al, 1988). By analogy, it might be expected that a similar analysis of breast tumors in different stages of progression would likewise show the accumulation of genetic changes with progression. However, genetic analysis of breast cancer specimens has suggested that the individual foci of tumor identifiable by microscopic examination may not show the precursor-product relationship often observed in colon cancer. Cytogenetic studies in particular have revealed sufficient genetic heterogeneity to lead some investigators to the conclusion that breast carcinomas commonly contain multiple genetically divergent clones of malignant cells, sometimes showing no evidence of a clonally related precursor (Niederacher et al, 1996; Wolman, 1986; Wolman and Heppner, 1992).

In this study a panel of breast carcinomas was analyzed to determine the extent of clonal divergence in tumor foci at progressive stages of tumor evolution. Specimens consisting of relatively pure populations of cells representing the various stages in the progression of the tumor were obtained by microdissection. Analysis of each microdissected specimen for genetic changes at multiple loci allowed an assessment of the clonal relationship between the distinct populations of tumor cells. Surprisingly, when distinct tumor components showed changes in the pattern of loss of heterozygosity ( $\mathrm{LOH}$ ) observed, more advanced tumor stages often could not be interpreted as clonal derivatives of earlier stages of the same tumor. Four of the 
cases demonstrating divergent clones were analyzed in greater detail by characterizing additional regions of the microdissected tumor and foci of morphologically benign epithelium. In all four cases, microdissected normal lobules demonstrated $\mathrm{LOH}$ at at least one locus, and the patterns of $\mathrm{LOH}$ in the tumor specimens revealed marked complexity in the pathways of tumor progression. The results demonstrate that genetically divergent clones commonly arise during breast cancer progression.

\section{Results}

Foci of intraductal, invasive, and metastatic carcinoma were identified and microdissected from 115 cases of breast cancer from the archives of the Armed Forces Institute of Pathology (Lichy et al, 1998). Each microdissected specimen was analyzed for $\mathrm{LOH}$ at a panel of microsatellite markers chosen from genetic loci previously shown to exhibit high frequency $\mathrm{LOH}$ in breast cancer (Aldaz et al, 1995; Brenner and Aldaz, 1997; Callahan et al, 1992). This panel included markers on chromosomes 3p, 9p, 11p, 13q, 16q, 17p, and $17 q$. When two tumor components showed loss of different alleles of a given marker, the LOH pattern was scored as discordant, and taken as evidence that the tumor components represented divergent clones rather than successive stages of progression. Such evidence of genetic heterogeneity was obtained in 24 cases (21\%; Table 1). Of these, 15 had progressed to the stage of lymph node metastasis, whereas 9 were node negative. In 17 cases, the infiltrating tumor showed evidence of divergence from the intraductal component. In 11 of the 15 node positive tumors in this group, the metastasis showed evidence of diver-

Table 1. Summary of Discordant LOH Results

\begin{tabular}{|c|c|c|c|c|}
\hline \multirow[b]{2}{*}{ Case } & \multirow[b]{2}{*}{ Specimens $^{a}$} & \multicolumn{2}{|c|}{ Discordant $\mathrm{LOH}$ at } & \multirow[b]{2}{*}{ p53 Status ${ }^{c}$} \\
\hline & & Locus & $\begin{array}{c}\text { Tumor } \\
\text { Components }^{b}\end{array}$ & \\
\hline 1 & ID, INF, MET & $17 p$ & $\mathrm{INF} \Leftrightarrow \mathrm{MET}$ & \\
\hline 6 & ID, INF & $\begin{array}{l}17 p \\
17 q\end{array}$ & $\begin{array}{l}\mathrm{ID} \Leftrightarrow \mathrm{INF} \\
\mathrm{ID} \Leftrightarrow \mathrm{INF}\end{array}$ & $\begin{array}{l}\text { ID: 228: GAC } \rightarrow \text { TAC } \\
\text { INF: WT }\end{array}$ \\
\hline 7 & ID, INF, MET & $16 q$ & $\mathrm{ID} \Leftrightarrow \mathrm{INF}$ & \\
\hline 16 & ID, INF, MET & $\begin{array}{l}11 p \\
16 q \\
17 p \\
17 q\end{array}$ & $\begin{array}{l}\text { INF } \Leftrightarrow \text { ID, MET } \\
\text { INF } \Leftrightarrow \text { MET } \\
\text { ID } \Leftrightarrow \text { INF, MET } \\
\text { INF } \Leftrightarrow \text { MET }\end{array}$ & WT \\
\hline 41 & ID, INF, MET & $\begin{array}{l}3 p \\
13 q\end{array}$ & $\begin{array}{l}\text { ID } \Leftrightarrow \text { INF, MET } \\
\text { ID } \Leftrightarrow \text { INF, MET }\end{array}$ & WT \\
\hline 43 & ID, INF & $\begin{array}{l}16 q \\
17 q\end{array}$ & $\begin{array}{l}I D \Leftrightarrow I N F \\
I D \Leftrightarrow I N F\end{array}$ & WT \\
\hline 49 & ID, INF, MET & $16 q$ & $\mathrm{INF} \Leftrightarrow \mathrm{MET}$ & \\
\hline 50 & ID, INF, MET & $17 q$ & $\mathrm{ID} \Leftrightarrow \mathrm{INF}, \mathrm{MET}$ & WT \\
\hline 52 & ID, INF & $17 p$ & $\mathrm{ID} \Leftrightarrow \mathrm{INF}$ & WT \\
\hline 53 & ID, INF & $17 p$ & $\mathrm{ID} \Leftrightarrow \mathrm{INF}$ & \\
\hline 61 & ID, INF, MET & $\begin{array}{l}11 p \\
17 q\end{array}$ & $\begin{array}{l}\text { INF } \Leftrightarrow \text { MET } \\
I D \Leftrightarrow I N F\end{array}$ & \\
\hline 64 & ID, INF, MET & $\begin{array}{l}17 p \\
17 q\end{array}$ & $\begin{array}{l}I D \Leftrightarrow I N F, M E T \\
I D \Leftrightarrow I N F, M E T\end{array}$ & $\begin{array}{l}\text { ID: 265: CTA } \\
\text { INF, Met: 265: CTG }\end{array}$ \\
\hline 66 & INF, MET & $\begin{array}{l}3 p \\
17 q\end{array}$ & $\begin{array}{l}\text { INF } \Leftrightarrow \text { MET } \\
\text { INF } \Leftrightarrow \text { MET }\end{array}$ & \\
\hline 78 & ID, INF, MET & $16 q$ & $\mathrm{ID}, \mathrm{INF} \Leftrightarrow \mathrm{MET}$ & \\
\hline 79 & ID, INF & $9 p$ & $\mathrm{ID} \Leftrightarrow \mathrm{INF}$ & \\
\hline 85 & INF, MET & $\begin{array}{l}13 q \\
17 p\end{array}$ & $\begin{array}{l}\text { INF } \Leftrightarrow \text { MET } \\
\text { INF } \Leftrightarrow \text { MET }\end{array}$ & \\
\hline 93 & ID, INF, MET & $17 p$ & INF $\Leftrightarrow$ MET & WT \\
\hline 101 & ID, INF & $17 q$ & $\mathrm{ID} \Leftrightarrow \mathrm{INF}$ & 286: GAA $\rightarrow$ AAA \\
\hline 106 & ID, INF, MET & $17 p$ & $\mathrm{ID} \Leftrightarrow \mathrm{INF}, \mathrm{MET}$ & WT \\
\hline 111 & ID, INF & $13 q$ & $\mathrm{ID} \Leftrightarrow \mathrm{INF}$ & \\
\hline 112 & INF, MET & $16 q$ & $\mathrm{INF} \Leftrightarrow \mathrm{MET}$ & \\
\hline 113 & ID, INF, MET & $13 q$ & $\mathrm{ID} \Leftrightarrow \mathrm{INF} \Leftrightarrow \mathrm{MET}$ & \\
\hline 115 & ID, INF & $17 q$ & $\mathrm{ID} \Leftrightarrow \mathrm{INF}$ & 175: $\mathrm{CGC} \rightarrow \mathrm{CAG}$ \\
\hline 118 & ID, INF, MET & $16 q$ & $\mathrm{ID} \Leftrightarrow \mathrm{INF} \Leftrightarrow \mathrm{MET}$ & \\
\hline
\end{tabular}

${ }^{a}$ ID, intraductal; INF, infiltrating; MET, metastatic tumor.

${ }^{b}$ The symbol " $\Leftrightarrow$ " indicates discordant LOH between the indicated specimens.

${ }^{c}$ Mutations designated by codon number, sequence change; altered base in bold. 
gence from the infiltrating tumor. Discordant LOH was observed at loci on one chromosomal arm in 16 cases, on two in 7 cases, and on four in 1 case. Evidence for genetic heterogeneity was most commonly observed with markers on 17p (10 cases), 17q (9 cases), and $16 q$ ( 7 cases), followed by $13 q$ ( 4 cases), and 11p ( 2 cases). The $3 p$ and $9 p$ markers revealed discordant $\mathrm{LOH}$ in one case each. Examples of discordance between infiltrating and metastatic tumor (Cases 1, 16, and 112) and between intraductal and infiltrating tumor (Cases 111, 113, and 118) are presented in Figure 1. In Cases 113 and 118, the metastatic tumor showed retention of heterozygosity, indicating genetic divergence from both the intraductal and infiltrating tumor components in these cases. Because the metastatic components of Cases 113 and 118 showed LOH with markers at $17 q$ and $11 p$, respectively, the observed retention of heterozygosity in these metastases was not due to contamination with benign cells.

To gain a greater understanding of the relationship between the morphologic and the genetic anatomy of each specimen, more extensive microdissections and genetic analyses were carried out on four of these cases. In the following discussion, the designations "allele A" and "allele B" refer to the alleles yielding the larger and smaller polymerase chain reaction (PCR) products, respectively. Normal components are designated "N," intraductal components "ID," invasive tumor "INF," and metastases "MET." Multiple foci representing the same stage of progression are distinguished by a numerical designation. The results presented for these cases are compatible with several possible pathways of tumor evolution. For each case, a diagram is presented that gives the least complex pathway of tumor progression compatible with the
$\mathrm{LOH}$ data. These diagrams are drawn based on the assumption that, whenever compatible with the data, an observed $\mathrm{LOH}$ event represents a single occurrence in the evolution of the tumor, so that tumor components with $\mathrm{LOH}$ at a common locus are postulated to share a common precursor. In some cases, sufficient material was available to allow the assessment of p53 mutations by sequencing. It would be unlikely for a specific p53 mutation to occur more than once during tumor development, whereas $\mathrm{LOH}$ at a specific locus might be more likely to occur multiple times. Therefore, the finding of common or divergent p53 mutations among the tumor components provides additional supportive evidence for the proposed pathways, as discussed below.

\section{Case 43}

Two normal tissue specimens, one a lymph node $(N)$, the other a morphologically normal terminal duct lobular unit (TDLU, N1) were available for analysis (Fig. 2). Tumor components included a focus of comedo type intraductal carcinoma and an adjacent area of infiltrating ductal carcinoma. Specimen N1 demonstrated $\mathrm{LOH}$ at $13 q$ and $17 p$. For the remaining lower allele, the products obtained with this specimen at the $17 p$ marker showed several bands larger than the major band detected with the normal control specimen. This pattern was interpreted as a variant of the normal stutter pattern generated from this allele, although the possibility of a novel allele representing microsatellite instability at this locus cannot be excluded.

The intraductal and infiltrating tumor specimens represented genetically divergent clones relative to this normal specimen, as evidenced by discordant

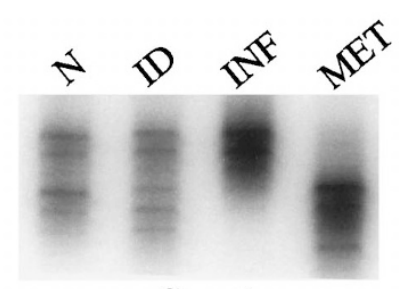

Case 1 D17S1880

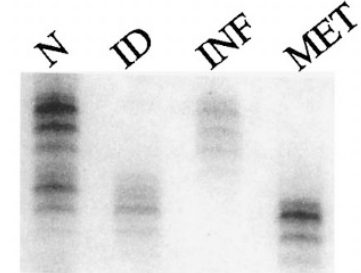

Case 16

D11S4046

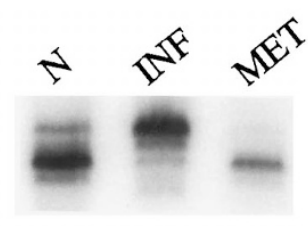

Case 112

D16S512

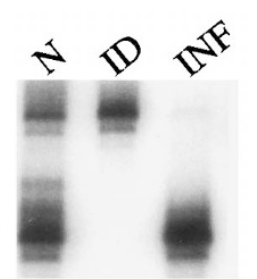

Case 111

D13S263

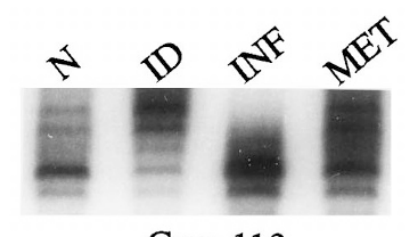

Case 113

D13S263

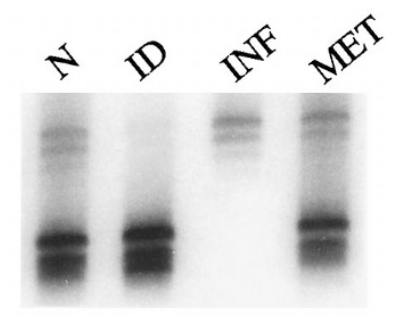

Case 118

\section{Figure 1.}

Examples of discordant loss of heterozygosity (LOH). N: Normal control (benign lymph node); ID: intraductal carcinoma; INF: infiltrating carcinoma; MET: metastatic tumor in lymph node. Case numbers correspond to those shown in Table 1. LOH results shown are for the indicated dinucleotide repeat polymorphism. Cases 1, 16, and 112 illustrate discordance between infiltrating and metastatic tumor foci. Cases 111, 113, and 118 illustrate discordance between intraductal and infiltrating tumor. 
A.

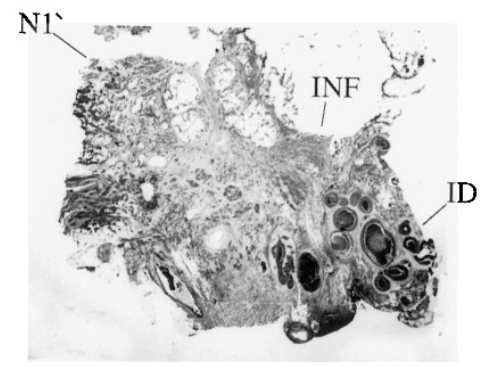

C.

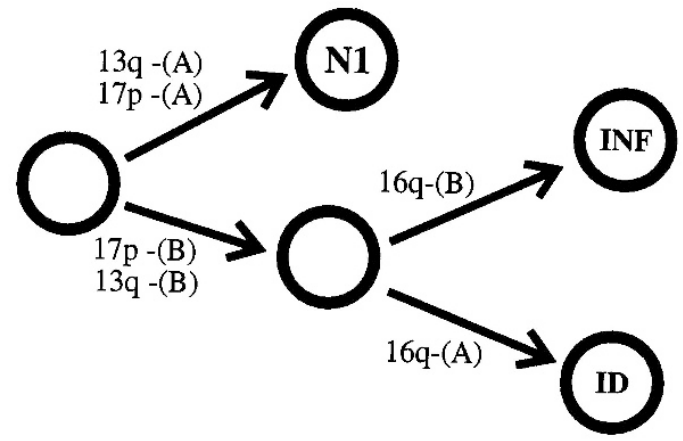

B.

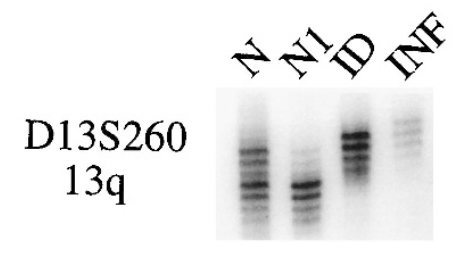

D17S831 $17 \mathrm{p}$

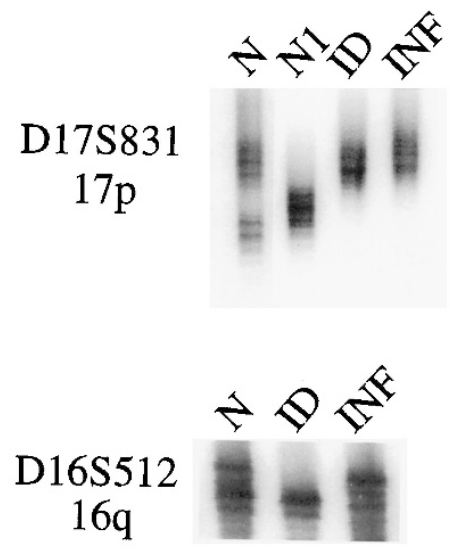

Figure 2.

Case 43. A, Histology: Low power view showing regions chosen for microdissection. N1: Benign terminal duct-lobular unit; ID: comedo type intraductal carcinoma; INF: Infiltrating tumor adjacent to ID; N: normal control prepared from a benign axillary lymph node. $B$, LOH data obtained at the indicated loci with lysates prepared from microdissected tumor components. The material available from specimen N1 was insufficient for LOH analysis at D16S512. C, Clonal relationship between microdissected tumor components suggested by $\mathrm{LOH}$ data. Open circles indicate a putative intermediate or precursor cell. $\mathrm{LOH}$ is indicated on the diagrams by chromosomal locus and the allele lost. Alleles are designated $(A)$ and $(B)$, where $(A)$ refers to the larger allele present in normal (lymph node) DNA. For example, $17 p-(B)$ means loss of the smaller allele for one of the 17p markers tested.

$\mathrm{LOH}$ at both of these loci. Thus, the malignant components appear to be clonally related to each other, but genetically distinct from the normal TDLU. Despite being clonally related, the intraductal and infiltrating tumor components diverge genetically relative to each other, as indicated by loss of opposite alleles at 16q. These results suggest that the two tumor components share a common precursor, indicated by the open circle in the diagram, which diverged along two distinct pathways, one developing into the intraductal lesion present in the surgical specimen, the other into an infiltrating carcinoma.

\section{Case 50}

Two morphologically normal TDLUs, labeled N1 and N2, were microdissected in addition to intraductal, invasive, and metastatic components. A benign lymph node served as the normal control. The LOH results imply that these specimens represent multiple divergent pathways of clonal evolution within and adjacent to the tumor (Fig. 3). Normal components N1 and N2 each contained multiple clonal abnormalities. Although these specimens showed loss of the same alleles at $17 p$ and $17 q$, suggesting a possible common precursor, these normal components demonstrated discordant allele loss at $11 \mathrm{p}$. With specimens N2 at $17 p$ and $\mathrm{N} 1$ at $17 \mathrm{q}$, the major PCR product for the remaining allele was shifted downward by one dinucleotide unit. These subtle changes were interpreted as variants of the stutter pattern for these alleles rather than as evidence of microsatellite instability. In contrast, the $3 p$ marker revealed several new bands common to N2 and INF but absent in the other specimens. These bands probably represent MSI at this locus. The new bands were unlikely to have resulted from contamination, because bands of this size were not present elsewhere on the autoradiogram, and amplification of these specimens with the other markers in our panel did not produce extra bands not present in the normal control. The diagram in Fig. 3 shows a pathway in which N2 and INF derive from a common precursor with $3 p \mathrm{MSI}$; however, the data are also compatible with the possibility that $\mathrm{N} 1$ and N2 represent divergent clones from a precursor with $17 p$ and $17 q \mathrm{LOH}$ and that the MSI at $3 p$ occurred independently in INF and N2.

The malignant components isolated from this specimen revealed patterns of $\mathrm{LOH}$ that implied genetic divergence from the adjacent morphologically normal epithelium. The intraductal tumor diverged from the other tumor components at the $17 p$ marker. The invasive and metastatic tumors show loss of common alleles on $17 p$ and $17 q$. Interestingly, the metastasis does not demonstrate the MSI 
A.

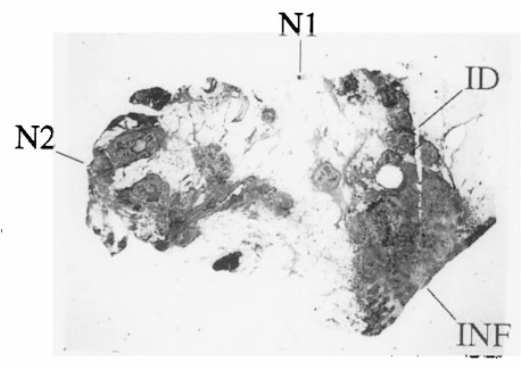

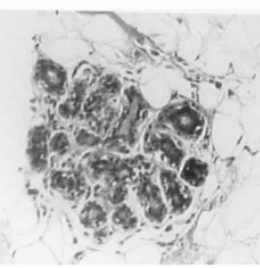

N1

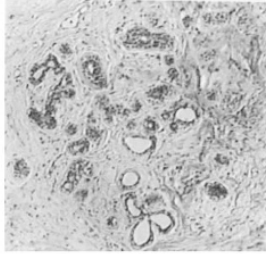

N2

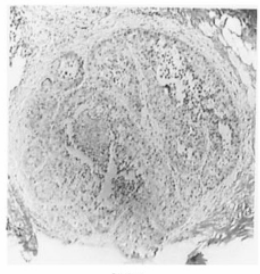

ID

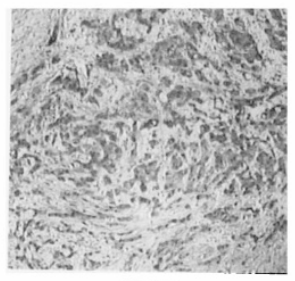

INF

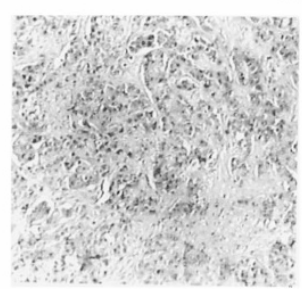

MET
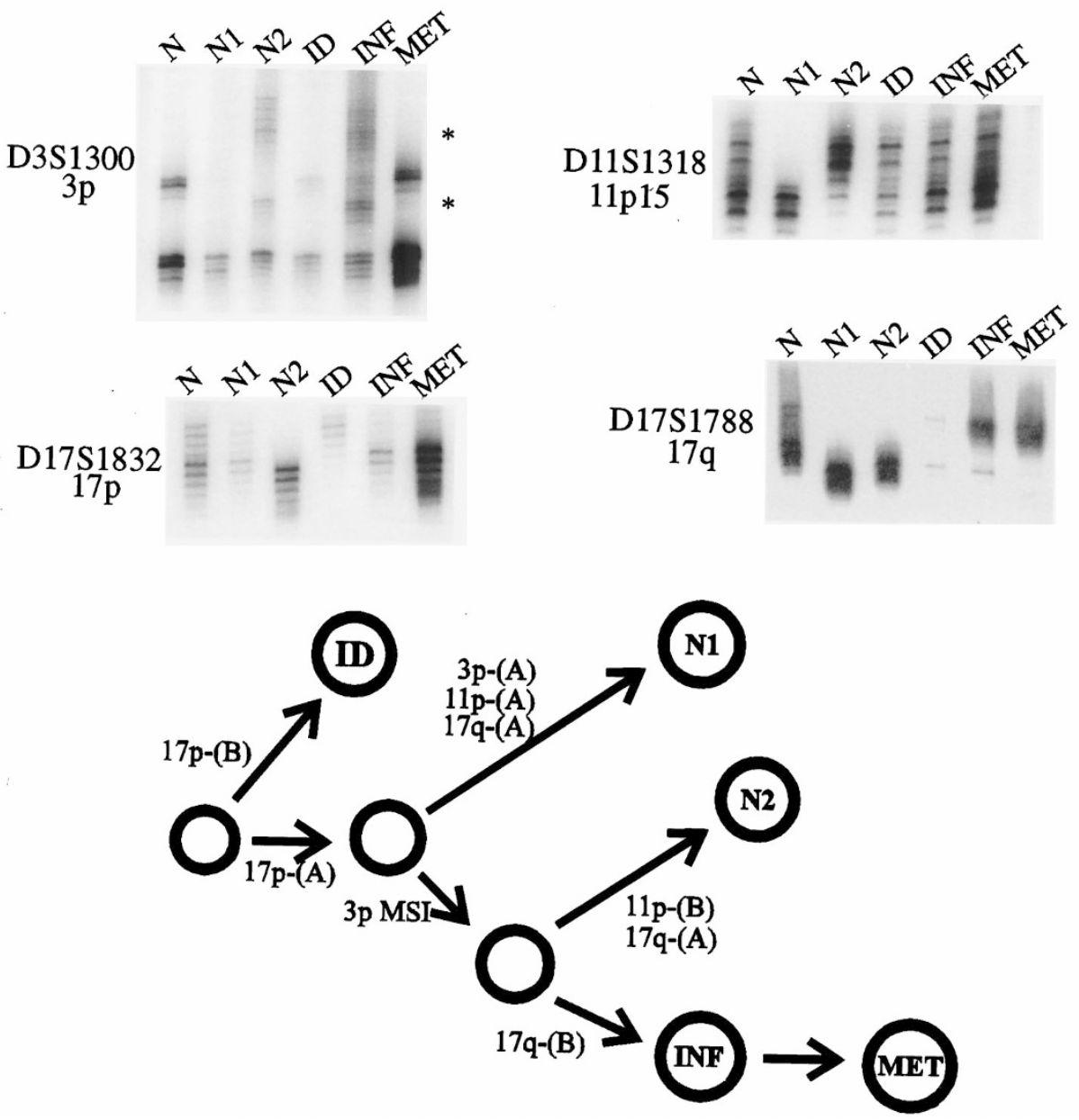

\section{Figure 3.}

Case 50. A, Histology: N1 and N2: Benign terminal duct-lobular units dissected from different regions of the specimen; ID: intraductal carcinoma; INF: infiltrating tumor adjacent to ID; N: benign axillary lymph node. $B, \mathrm{LOH}$ data obtained at the indicated loci. $C$, Clonal relationship between microdissected tumor components suggested by LOH data. Designation of alleles and LOH results is as in Figure 2; MSI: microsatellite instability, indicates the presence of one or more new alleles (marked with an asterisk at side of gel image) not detected in the normal specimen.

detected at $3 p$ in the invasive component. This suggests that one of several clones present in the invasive tumor, and in particular one that lacks the new allele resulting from microsatellite instability, gave rise to the metastatic tumor.

\section{Case 101}

A normal lobule (N1) was located $5 \mathrm{~mm}$ from a focus (ID1) of lobular carcinoma in situ (LCIS), which in turn was located $4 \mathrm{~mm}$ from a second focus of LCIS (ID2). 
ID2 was immediately adjacent to an area of infiltrating lobular carcinoma (INF). Despite the proximity of these lesions, substantial heterogeneity was observed among the four microdissected specimens. The pattern of LOH suggests two branches of clonal evolution, one leading to clones N1 and ID1, the other to ID2 and INF. N1 and ID1 both show LOH of allele A with a $9 p$ marker, consistent with the possibility of a common precursor. However, these specimens display discordant LOH at both 11p and 17q. ID1 demonstrates one additional abnormality, $\mathrm{LOH}$ at 13q. Specimens ID2 and INF share loss of allele B at $9 p$ and concomitant acquisition of a common new allele at 9p. Also consistent with a common precursor is the observation that these two specimens yielded results with D13S260 suggestive of apparent partial loss of allele A (a result that could be due to duplication of allele $B$ ). However, ID2 and INF demonstrated discordant LOH at $17 \mathrm{q}$, indicating that ID2 can not be interpreted simply as a precursor of INF, but that these specimens must represent divergent clones.

\section{Case 106}

Two benign TDLUs, two foci of intraductal carcinoma, and one each of infiltrating and metastatic tumor were analyzed (Fig. 4). A common clonal origin for normal specimens N1 and N2 was suggested by loss of the same allele at $17 q$ and by the presence of a common new allele at $11 p$. However, these normal specimens diverge genetically, with $\mathrm{LOH}$ at $17 \mathrm{p}$ in $\mathrm{N} 1$ and at $11 \mathrm{p}$ in N2. Although N1 and N2 did not share alleles in common with the lymph node specimen at the $11 \mathrm{p} 15$ marker, the possibility of a specimen mix-up was excluded by the data obtained with the other markers in the panel, including the $17 p$ and $17 q$ markers illustrated. None of the malignant specimens showed $\mathrm{LOH}$ at $17 \mathrm{q}$, suggesting a completely different path of evolution from the benign foci studied. The two intraductal lesions, ID1 and ID2, lost different alleles of the $17 \mathrm{p}$ marker. The metastasis could be a clonal derivative of ID2, as suggested by loss of a common allele at $17 \mathrm{p}$, or could have arisen from the invasive tumor by a second $\mathrm{LOH}$ event at this locus.

Assessment of Cases for Microsatellite Instability Phenotype and p53 Mutation. A Replication Error phenotype, often designated "RER+," has been described in genetic studies of colon cancer. This phenotype results in a very high frequency of MSI at short tandem repeat loci. In particular, the RER+ phenotype has been shown to correlate strongly with MSI at the single locus BAT26 (Hoang et al, 1997). The lysates from the full panel of 115 cases (Lichy et al, 1998) were tested for MSI at the BAT26 locus. All but one of the cases demonstrated the near homozygous genotype observed in most individuals. MSI at this locus was observed in only one tumor component in one case. Therefore, although MSI was observed sporadically in

A.

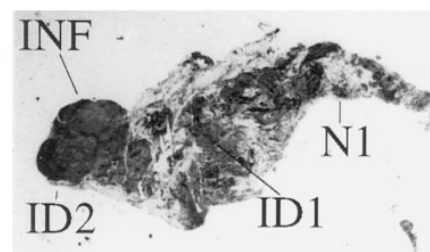

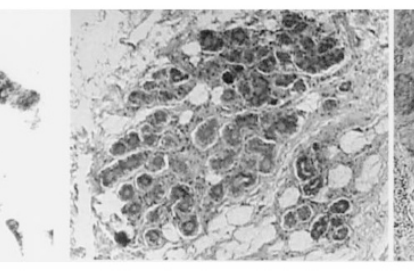

N1

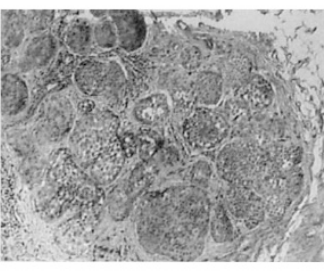

ID1

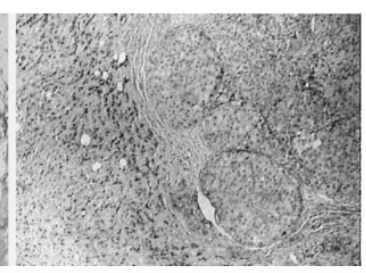

ID2\&INF
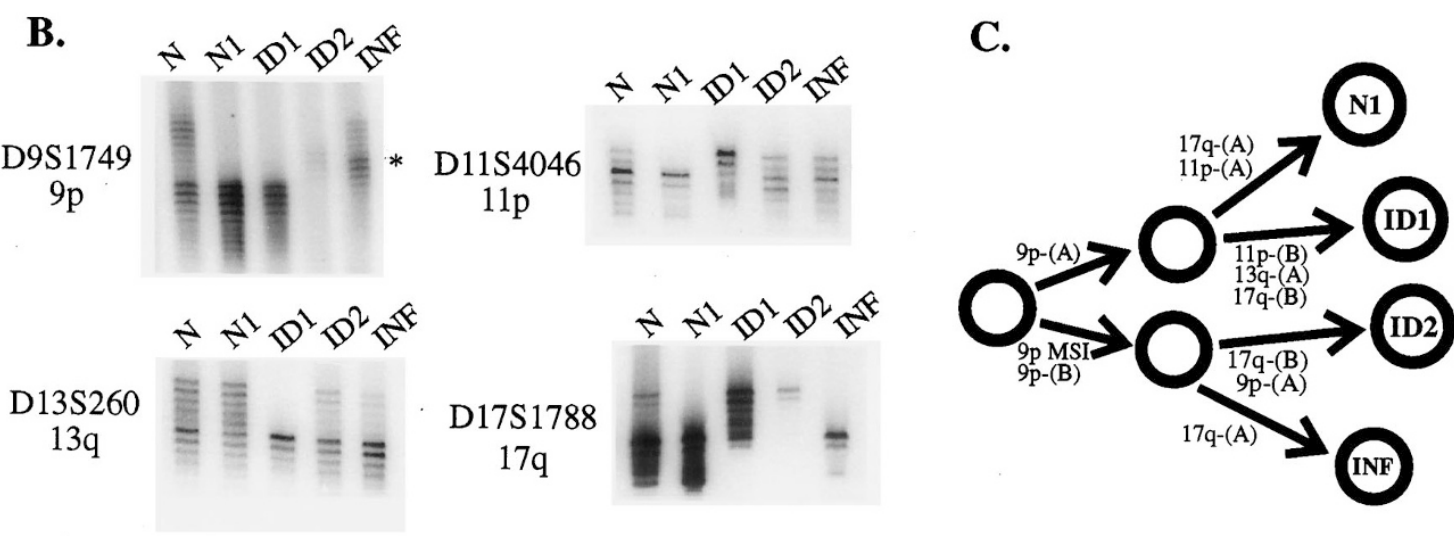

\section{Figure 4.}

Case 101. A, Histology: N1: Benign terminal duct-lobular unit; ID1: Iobular carcinoma in situ; ID2: Distinct focus of lobular carcinoma in situ; INF: infiltrating lobular carcinoma adjacent to ID2; N: benign axillary lymph node. B, LOH data obtained at the indicated loci. C, Clonal relationship between microdissected tumor components suggested by $\mathrm{LOH}$ data. Designation of alleles and $\mathrm{LOH}$ results is as in previous figures. 
these cases, there was no evidence for the RER+ phenotype.

Genetic instability in breast cancer has been associated with mutations of the p53 tumor suppressor gene, (Eyfjord et al, 1995a; 1995b) although some studies have not found this association (Deng et al, 1994). To determine if discordant LOH might reflect the presence of a p53 mutation, exons 5-8 of the p53 gene were sequenced in the normal tissue and tumor components of 11 cases with discordant $\mathrm{LOH}$ for which sufficient material was available. These exons contain approximately $80 \%$ of the p53 mutations in breast cancer (Casey et al, 1996). A p53 mutation was observed in three of these cases (Table 1). Of particular interest were Cases 6 and 64. In Case 6, a p53 mutation was detected in the intraductal tumor but not in the invasive or metastatic components. In Case 64, a single nucleotide polymorphism identified at codon 265 showed loss of opposite alleles in the intraductal and infiltrating tumor. These results corroborate the finding of discordant $\mathrm{LOH}$ for $17 \mathrm{p}$ markers in both of these cases and provide additional genetic evidence for distinct patterns of clonal evolution during histologic progression in a subset of breast carcinomas.

\section{Discussion}

Our observations indicate that the in situ, infiltrating, and metastatic components observed in surgically resected breast cancer specimens can in some cases represent genetically divergent clones rather than progressive stages in tumor progression. Of 115 cases analyzed, 24 (21\%) showed evidence of genetically divergent clones during progression from the intraductal to the metastatic stage. A more detailed microdissection of four of these cases revealed an even higher degree of genetic divergence among foci of tumor at various stages of progression. In all four of these cases, LOH was also detected in morphologically benign TDLUs adjacent to the tumor, indicating that normal lobules from biopsy specimens can contain clonal genetic lesions.

Several studies, based on cytogenetic methods, comparative genomic hybridization, and $\mathrm{LOH}$ analysis, have reported observations of genetically divergent clones in breast cancer cells, their presumed precursors, or in normal breast epithelium. In one study, LOH was identified in normal TDLUs adjacent to tumor (Deng et al, 1996), but genetically divergent clones were not identified. The results shown here demonstrate that the genetic changes present in TDLUs are not necessarily those present in the tumor. Rather than being a precursor to the tumor, such TDLUs must represent divergent pathways of clonal evolution. Genetic heterogeneity has been previously demonstrated in different foci of intraductal carcinoma microdissected from the same specimen (Fujii et al, 1996). In that study, heterogeneity among invasive or metastatic components appeared to be rare, and all clones from any given tumor sample could be linked to a common precursor by loss of similar genetic markers. Another study, using comparative genomic hy- bridization, demonstrated that asynchronous breast cancer metastases frequently have little evidence of clonal relatedness to the primary tumor (Kuukasjarvi et al, 1997). Cytogenetic analysis of tumor specimens grown for short periods of time in culture has provided evidence for the presence of apparently unrelated clones in half of the tumors analyzed (Heim et al, 1997; Pandis et al, 1993; Teixeira et al, 1994; 1995; 1996; 1997). Clonal chromosome aberrations have also been reported in benign lesions including fibroadenoma, atypical hyperplasia, and intraductal papilloma (Dietrich et al, 1995; Kasami et al, 1997; Petersson et al, 1997; Rosenberg et al, 1996) as well as in benign epithelium in patients from breast cancer families (Petersson et al, 1996) or at no increased risk of breast cancer (Larson et al, 1998). Our results, in agreement with these studies, support the idea that formation of genetically divergent clones can occur in benign breast epithelium showing no morphologic abnormalities. We have further shown that individual tumor foci representing different stages of progression can contain dominant clones that have diverged genetically from other regions of the tumor. Our results also demonstrate that this divergence does not occur exclusively among multiple intraductal foci or asynchronous metastases, but also can be detected during progression from intraductal to infiltrating and from infiltrating to synchronous metastatic tumor.

It is interesting to interpret the finding of genetic heterogeneity in breast cancers in light of the two-hit model of tumor suppressor gene inactivation (Knudson, 1971; 1985). According to this model, both copies of a tumor suppressor gene must be inactivated to eliminate the function of that gene. In the classic case of familial retinoblastoma, one copy of the $\mathrm{Rb}$ gene is mutationally inactivated in the germline. In sporadic retinoblastoma, both copies of the $\mathrm{Rb}$ gene must be inactivated somatically. Similarly, loci showing high frequency LOH in breast cancer, such as those analyzed in this study, are thought to contain tumor suppressor genes important for the development of the cancer. The phenomenon of $\mathrm{LOH}$ is typically interpreted as one of the two hits necessary for functional inactivation of these tumor suppressor genes. Theoretically, either of the two hits-the mutation or the deletion-could occur first and not affect the phenotype of the cell. Therefore, it may be that in breast epithelium, LOH can occur sporadically during normal development without leading to any phenotypic abnormalities. A recent study reporting $\mathrm{LOH}$ in ductal or lobular epithelium microdissected from a small number of benign breasts supports this hypothesis (Larson et al, 1998). However, the presence of $\mathrm{LOH}$ in normal breast epithelium may constitute a predisposing factor for the subsequent development of carcinoma. It remains to be determined whether the presence of LOH in benign breast tissue constitutes a physiologic alteration in genome anatomy that occurs universally during the development of the breast, or whether this phenomenon occurs in only a subset of individuals, and if the latter is the case, whether such 
individuals have a greater relative risk for the development of breast cancer.

We studied cases showing discordant LOH for two types of genetic abnormalities associated with deficiencies in maintaining chromosomal integrity. Instability at the BAT26 locus has been found to be a sensitive indicator of the replication error, or RER+, phenotype in colon cancer (Hoang et al, 1997). Using this assay, we found no evidence of a microsatellite instability phenotype in breast cancer, although sporadic examples of microsatellite instability were observed. Thus, these examples most likely represent a "baseline" level of instability rather than the RER+ phenotype described in colon cancer. These observations are compatible with most reports in the literature, which find some sporadic $\mathrm{MSI}$ in microdissected breast cancer, but do not find the high frequency MSI observed in colon cancers with the RER + phenotype (Aldaz et al, 1995; Contegiacomo et al, 1995; Dillon et al, 1997; Formantici et al, 1999; Fujii et al, 1998; Gorgoulis et al, 1998; Kasami et al, 1997; Paulson et al, 1996; Rush et al, 1997; Shaw et al, 1996; Sourvinos et al, 1997; Tomita et al, 1999; Toyama et al, 1996; Walsh et al, 1998; Wooster et al, 1994; Yee et al, 1994). One recent study (Anbazhagan et al, 1999) reported no examples of sporadic MSI in a large panel of breast cancers and other studies have observed
MSI only rarely ( $<5 \%$ of cases) (Huiping et al, 1999; Jonsson et al, 1995). The reason for the discrepancies in observed $\mathrm{MSI}$ rates in breast cancer is unclear but may be related to the methods of microdissection employed. Foci with MSI may only become evident when very small foci, such as individual TDLUs, are examined, as was done in the work shown in Figs. 2-5. If multiple morphologically similar foci, areas of intraductal carcinoma for example, are combined into one specimen, MSI limited to only one or a few such foci would most likely be missed.

Despite limiting amounts of material, it was possible to obtain the sequence of p53 exons 5-8 from 11 of the cases with discordant $\mathrm{LOH}$. Mutations were detected in three $(27 \%)$ of these cases. This percentage is similar to the frequency of p53 mutations usually reported in breast cancer, and therefore does not suggest that a p53 mutation results in a higher rate of genetic heterogeneity. It has been proposed that tumors showing $\mathrm{LOH}$ at multiple markers have a genetically determined deficiency in chromosomal maintenance (Cahill et al, 1998). In support of this concept, two of 19 colorectal carcinoma cell lines with a "Chromosomal Instability" phenotype were shown to contain mutations in the gene BUB1, the human homolog of a yeast gene involved in maintaining chromosomal integrity. It will be of interest to deter-

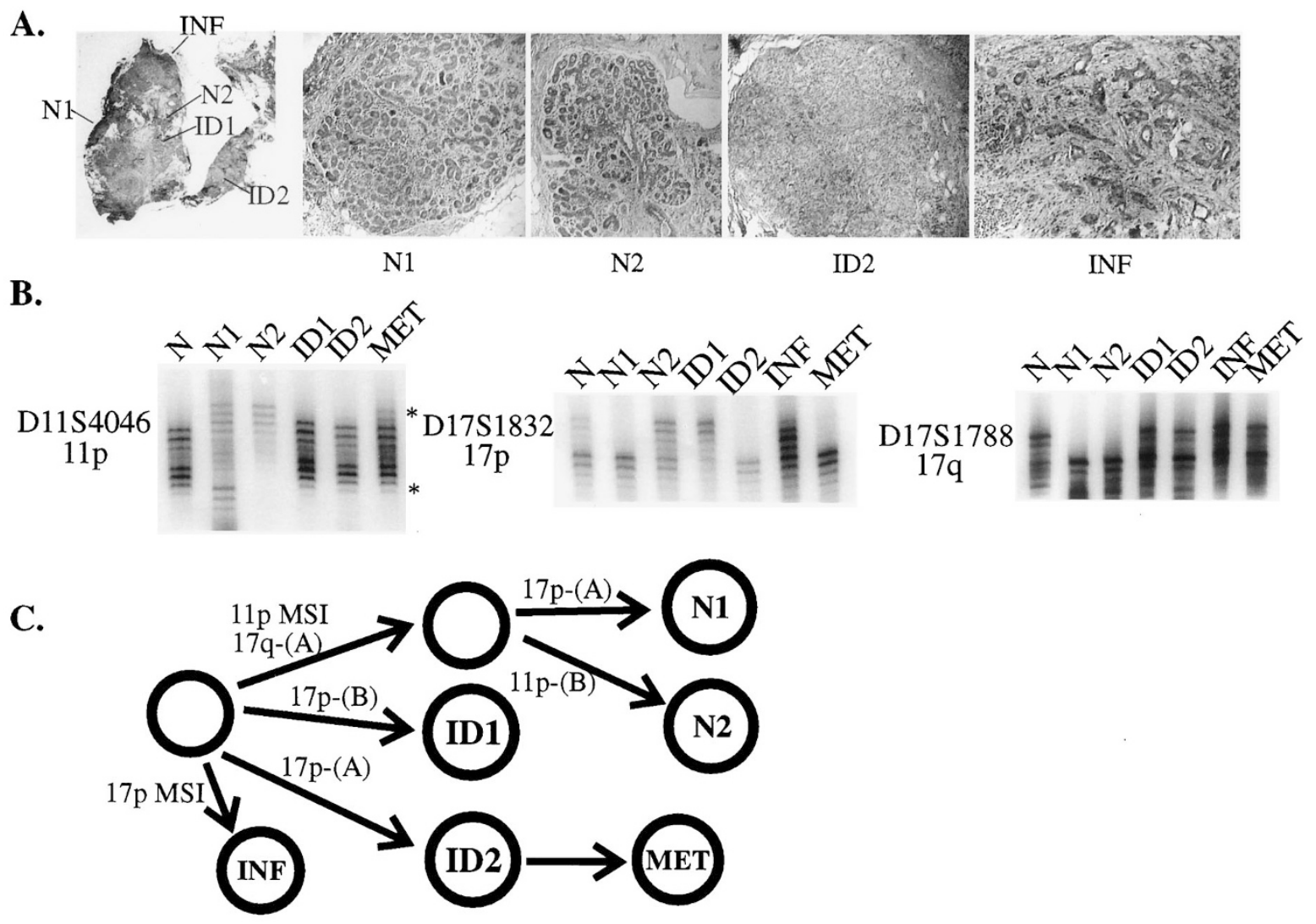

Figure 5.

Case 106. A, Histology: N1 and N2: Benign terminal duct-Iobular units; ID1 and ID2: intraductal carcinoma; INF: infiltrating carcinoma; N: benign axillary lymph node. $B$, LOH data obtained at the indicated loci. The material available from the infiltrating tumor was insufficient for LOH testing at D11S4046. $C$, Clonal relationship between microdissected tumor components suggested by $\mathrm{LOH}$ data. Designation of alleles and LOH results is as in previous figures. 
mine whether breast tumors containing high frequency $\mathrm{LOH}$ or genetic heterogeneity have mutations in such genes, and if so, whether these mutations are present in the germline or develop during the early stages of carcinogenesis.

In addition to findings such as those reported here for breast cancer, genetic heterogeneity has been reported in studies of microdissected prostate cancer. Analysis of $\mathrm{LOH}$ in physically separate tumor nodules was consistent with an independent origin in 15 of 18 informative cases (Cheng et al, 1998). Characterization of mutations in the PTEN/MMAC1 tumor suppressor gene in multiple metastatic lesions from individual patients led to the conclusion that the metastases were often heterogeneous at the genetic level (Suzuki et al, 1998). A microdissection and $\mathrm{LOH}$ approach similar to that applied in the present study revealed a high incidence of genetic heterogeneity among distinct foci of prostate carcinoma (Macintosh et al, 1998).

The ability to identify genetic markers in solid tumors, such as point mutations and loss of heterozygosity ( $\mathrm{LOH})$, has potential clinical utility in assays to detect small populations of malignant cells in blood or tissue specimens. Strategies for the design of such assays often rely on the assumption that an individual tumor is clonal with respect to a specific mutation. The data reported here demonstrate that this assumption is not always correct. The results indicate that the different tumor components observed microscopically in breast cancer specimens commonly represent genetically divergent clones.

\section{Materials and Methods}

\section{Microdissection}

The cases used in this study were obtained from the archives of the Armed Forces Institute of Pathology, Washington, DC, as previously described (Lichy et al, 1998). Case numbers used here correspond to the case numbers in the previous study. To identify individual tumor components, $12 \mu \mathrm{m}$ sections were cut from the blocks and deparaffinized with Hemo-De (Fisher Scientific, Pittsburgh, Pennsylvania). Tumor components were identified and dissected from surrounding tissue with a $21 \mathrm{G}$ needle under direct microscopic observation. For most cases, a benign lymph node was available for use as the normal DNA control. The dissected tissue was placed directly in lysis buffer, containing nonionic detergents and Proteinase $\mathrm{K}$ and incubated for $16-20$ hours at $55^{\circ} \mathrm{C}$ followed by 5 minutes at $95^{\circ} \mathrm{C}$ to inactivate the protease. Insoluble material was removed by centrifugation and the cleared lysate was used as the source of genomic DNA for PCR.

\section{Markers for LOH Analysis}

The panel of markers was chosen to represent loci that show frequent $\mathrm{LOH}$ in breast cancer. The markers used and their associated tumor suppressor genes, when known, included the following: chromosome 3p:
D3S1300 (FHIT gene) and C3-CA373; chromosome 9p21 (p16 cdk inhibitor): D9S1748 and D9S1749; chromosome 11p15: D11S4046, D11S1318, and TH; chromosome 13q: D13S260 and D13S263 (RB and BRCA2 genes); chromosome 16q: D16S496, D16S421, and D16S512; chromosome 17p: TP53, D17S1832, and D17S1880; and chromosome 17q: D17S1788, D17S795, D17S855 (BRCA1), D17S183, and D17S1830. PCR reactions were carried out with one primer end-labeled with ${ }^{32} \mathrm{P}$. Products were analyzed on standard denaturing sequencing gels and detected by autoradiography using Kodak XAR5 film (Kodak, Rochester, New York). For quantitation of band intensities, dried gels were exposed to storage phosphor screens that were scanned with a Molecular Dynamics Storm imaging system. A result was scored as $\mathrm{LOH}$ if there was a reduction in allele ratio of greater than $50 \%$ in the tumor relative to the normal control DNA. The presence of a new allele in a tumor specimen not seen in the normal control was interpreted as microsatellite instability (MSI). Two patterns of $\mathrm{LOH}$ were scored as "discordant:" (a) LOH in one tumor component together with retention of heterozygosity in a specimen representing a later stage of progression; and (b) LOH of opposite alleles in two specimens from the same tumor.

Several precautions were taken to guard against artifacts due to PCR contamination. Negative controls containing no tissue lysate were included with each PCR set-up. The discordant results shown in Fig. 1 were observed reproducibly with two independently microdissected specimens. For the more detailed analysis presented in Figs. 2-5, fresh sections were cut and microdissected. Results with the second sets of lysates reproduced those obtained with the initial specimens with the exception that only the initial preparation of the infiltrating component of Case 50 showed $\mathrm{LOH}$ at $11 \mathrm{p} 15$. This discrepancy probably resulted from the dissection of distinct foci of infiltrating tumor for the two specimens. Some of these microdissected specimens contained sufficient material to permit analysis with only a subset of the genetic markers. In cases showing novel alleles, interpreted as $\mathrm{MSI}$, the novel allele was observed reproducibly in different specimens from the same case and differed in size from bands generated with other cases in our panel. These observations argue against an artifactual origin for the novel alleles illustrated in the figures.

\section{p53 Sequencing}

Partial sequencing of the p53 gene from 11 cases was carried out as previously described (Przygodzki et al, 1996). Exons 5, 7, and 8 were amplified in PCR reactions containing primers specific for each exon. PCR products were gel purified and the sequence was determined by cycle sequencing using fluorescent dye terminators followed by electrophoresis and detection on a Perkin-Elmer Model 370 (Perkin-Elmer Cetus, Norwalk, Connecticut) automated DNA sequencer. 


\section{Acknowledgements}

We are grateful for the assistance of Annette Geissel in cutting blocks and preparing histologic sections.

\section{References}

Aldaz CM, Chen T, Sahin A, Cunningham J, and Bondy M (1995). Comparative allelotype of in situ and invasive human breast cancer: High frequency of microsatellite instability in lobular breast carcinomas. Cancer Res 55:3976-3981.

Anbazhagan R, Fujii H, and Gabrielson E (1999). Microsatellite instability is uncommon in breast cancer. Clin Cancer Res 5:839-844.

Boland CR, Sato J, Appelman HD, Bresalier RS, and Feinberg AP (1995). Microallelotyping defines the sequence and tempo of allelic losses at tumour suppressor gene loci during colorectal cancer progression. Nat Med 1:902-909.

Brenner AJ and Aldaz CM (1997). The genetics of sporadic breast cancer. Prog Clin Biol Res 396:63-82.

Cahill DP, Lengauer C, Yu J, Riggins GJ, Willson JK, Markowitz SD, Kinzler KW, and Vogelstein B (1998). Mutations of mitotic checkpoint genes in human cancers [See comments]. Nature 392:300-303.

Callahan R, Cropp CS, Merlo GR, Liscia DS, Cappa AP, and Lidereau R (1992). Somatic mutations and human breast cancer. A status report. Cancer 69:1582-1588.

Casey G, Lopez ME, Ramos JC, Plummer SJ, Arboleda MJ, Shaughnessy M, Karlan B, and Slamon DJ (1996). DNA sequence analysis of exons 2 through 11 and immunohistochemical staining are required to detect all known p53 alterations in human malignancies. Oncogene 13:1971-1981.

Cheng L, Song SY, Pretlow TG, Abdul-Karim FW, Kung HJ, Dawson DV, Park WS, Moon YW, Tsai ML, Linehan WM, Emmert-Buck MR, Liotta LA, and Zhuang Z (1998). Evidence of independent origin of multiple tumors from patients with prostate cancer. J Natl Cancer Inst 90:233-237.

Contegiacomo A, Palmirotta R, De Marchis L, Pizzi C, Mastranzo P, Delrio P, Petrella G, Figliolini M, Bianco AR, Frati L, Cama A, and Mariani-Costantini R (1995). Microsatellite instability and pathological aspects of breast cancer. Int $\mathrm{J}$ Cancer 64:264-268.

Deng G, Chen LC, Schott DR, Thor A, Bhargava V, Ljung BM, Chew K, and Smith HS (1994). Loss of heterozygosity and p53 gene mutations in breast cancer. Cancer Res 54:499505.

Deng G, Lu Y, Zlotnikov G, Thor AD, and Smith HS (1996). Loss of heterozygosity in normal tissue adjacent to breast carcinomas. Science 274:2057-2059.

Dietrich CU, Pandis N, Teixeira MR, Bardi G, Gerdes AM, Andersen JA, and Heim S (1995). Chromosome abnormalities in benign hyperproliferative disorders of epithelial and stromal breast tissue. Int J Cancer 60:49-53.

Dillon EK, de Boer WB, Papadimitriou JM, and Turbett GR (1997). Microsatellite instability and loss of heterozygosity in mammary carcinoma and its probable precursors. $\mathrm{Br} \mathrm{J}$ Cancer 76:156-162.

Eyfjord JE, Thorlacius S, Steinarsdottir M, Valgardsdottir R, Ogmundsdottir HM, and Anamthawat-Jonsson K (1995a). p53 abnormalities and genomic instability in primary human breast carcinomas. Cancer Res 55:646-651.
Eyfjord JE, Thorlacius S, Valgardsdottir R, Gretarsdottir S, Steinarsdottir M, and Anamthawat-Jonsson K (1995b). TP53 abnormalities and genetic instability in breast cancer. Acta Oncol 34:663-667.

Fearon ER and Vogelstein B (1990). A genetic model for colorectal tumorigenesis. Cell 61:759-767.

Formantici C, Orlandi R, Ronchini C, Pilotti S, Ranzani GN, Colnaghi MI, and Menard S (1999). Absence of microsatellite instability in breast carcinomas with both p53 and c-erbB-2 alterations. J Pathol 187:424-427.

Fujii H, Marsh C, Cairns P, Sidransky D, and Gabrielson E (1996). Genetic divergence in the clonal evolution of breast cancer. Cancer Res 56:1493-1497.

Fujii S, Takeshima Y, Arihiro K, Kaneko M, and Inai K (1998). Microsatellite instability in breast cancers with special reference to patients' age and bilaterality. Hiroshima J Med Sci 47:89-97.

Gorgoulis VG, Koutroumbi EN, Kotsinas A, Zacharatos P, Markopoulos C, Giannikos L, Kyriakou V, Voulgaris Z, Gogas I, and Kittas C (1998). Alterations of p16-pRb pathway and chromosome locus 9p21-22 in sporadic invasive breast carcinomas. Mol Med 4:807-822.

Heim S, Teixeira MR, Dietrich CU, and Pandis N (1997). Cytogenetic polyclonality in tumors of the breast. Cancer Genet Cytogenet 95:16-19.

Hoang JM, Cottu PH, Thuille B, Salmon RJ, Thomas G, and Hamelin R (1997). BAT-26, an indicator of the replication error phenotype in colorectal cancers and cell lines. Cancer Res 57:300-303.

Huiping C, Johannsdottir JT, Arason A, Olafsdottir GH, Eiriksdottir G, Egilsson V, and Ingvarsson S (1999). Replication error in human breast cancer: Comparison with clinical variables and family history of cancer. Oncol Rep 6:117-122.

Jonsson M, Johannsson O, and Borg A (1995). Infrequent occurrence of microsatellite instability in sporadic and familial breast cancer. Eur J Cancer 31A:2330-A2334.

Kasami M, Vnencak-Jones CL, Manning S, Dupont WD, and Page DL (1997). Loss of heterozygosity and microsatellite instability in breast hyperplasia. No obligate correlation of these genetic alterations with subsequent malignancy. Am J Pathol 150:1925-1932.

Knudson AG (1971). Mutation and cancer: statistical study of retinoblastoma. Proc Natl Acad Sci USA 68:820-824.

Knudson AG, Jr (1985). Hereditary cancer, oncogenes, and antioncogenes. Cancer Res 45:1437-1443.

Kuukasjarvi T, Karhu R, Tanner M, Kahkonen M, Schaffer A, Nupponen N, Pennanen S, Kallioniemi A, Kallioniemi OP, and Isola J (1997). Genetic heterogeneity and clonal evolution underlying development of asynchronous metastasis in human breast cancer. Cancer Res 57:1597-1604.

Larson PS, de las Morenas A, Cupples LA, Huang K, and Rosenberg CL (1998). Genetically abnormal clones in histologically normal breast tissue. Am J Pathol 152:1591-1598.

Lichy J, Zavar M, Tsai M, O'Leary T, and Taubenberger J (1998). Loss of heterozygosity at chromosome 11p15 during histological progression of ductal carcinoma of the breast. Am J Path.

Macintosh CA, Stower M, Reid N, and Maitland NJ (1998). Precise microdissection of human prostate cancers reveals genotypic heterogeneity. Cancer Res 58:23-28. 
Niederacher D, Schnurch HG, An HX, Ellenberger I, Dall P, van Roeyen CR, Kuppers V, and Beckmann MW (1996). Detection of sequential genetic alterations relevant for breast cancer development. Eur J Cancer Prev 5:497-503.

Nowell PC (1976). The clonal evolution of tumor cell populations. Science 194:23-28.

Pandis N, Heim S, Bardi G, Idvall I, Mandahl N, and Mitelman F (1993). Chromosome analysis of 20 breast carcinomas: cytogenetic multiclonality and karyotypic-pathologic correlations. Genes Chromosomes Cancer 6:51-57.

Paulson TG, Wright FA, Parker BA, Russack V, and Wahl GM (1996). Microsatellite instability correlates with reduced survival and poor disease prognosis in breast cancer. Cancer Res 56:4021-4026.

Petersson C, Pandis N, Mertens F, Adeyinka A, Ingvar C, Ringberg A, Idvall I, Bondeson L, Borg A, Olsson H, Kristoffersson U, and Mitelman F (1996). Chromosome aberrations in prophylactic mastectomies from women belonging to breast cancer families. Genes Chromosomes Cancer 16: 185-188.

Petersson C, Pandis N, Rizou H, Mertens F, Dietrich CU, Adeyinka A, Idvall I, Bondeson L, Georgiou G, Ingvar C, Heim S, and Mitelman F (1997). Karyotypic abnormalities in fibroadenomas of the breast. Int $\mathrm{J}$ Cancer 70:282-286.

Przygodzki RM, Finkelstein SD, Langer JC, Swalsky PA, Fishback N, Bakker A, Guinee DG, Koss M, and Travis WD (1996). Analysis of p53, K-ras-2, and C-raf-1 in pulmonary neuroendocrine tumors. Correlation with histological subtype and clinical outcome. Am J Pathol 148:1531-1541.

Rosenberg CL, de las Morenas A, Huang K, Cupples LA, Faller DV, and Larson PS (1996). Detection of monoclonal microsatellite alterations in atypical breast hyperplasia. J Clin Invest 98:1095-1100.

Rush EB, Calvano JE, van Zee KJ, Zelenetz AD, and Borgen PI (1997). Microsatellite instability in breast cancer. Ann Surg Oncol 4:310-315.

Shaw JA, Walsh T, Chappell SA, Carey N, Johnson K, and Walker RA (1996). Microsatellite instability in early sporadic breast cancer. Br J Cancer 73:1393-1397.

Sourvinos G, Kiaris H, Tsikkinis A, Vassilaros S, and Spandidos DA (1997). Microsatellite instability and loss of heterozygosity in primary breast tumours. Tumour Biol 18:157166.

Suzuki H, Freije D, Nusskern DR, Okami K, Cairns P, Sidransky D, Isaacs WB, and Bova GS (1998). Interfocal heterogeneity of PTEN/MMAC1 gene alterations in multiple metastatic prostate cancer tissues. Cancer Res 58:204-209.

Teixeira MR, Pandis N, Bardi G, Andersen JA, and Heim S (1996). Karyotypic comparisons of multiple tumorous and macroscopically normal surrounding tissue samples from patients with breast cancer. Cancer Res 56:855-859.
Teixeira MR, Pandis N, Bardi G, Andersen JA, Bohler PJ, Qvist H, and Heim S (1997). Discrimination between multicentric and multifocal breast carcinoma by cytogenetic investigation of macroscopically distinct ipsilateral lesions. Genes Chromosomes Cancer 18:170-174.

Teixeira MR, Pandis N, Bardi G, Andersen JA, Mandahl N, Mitelman F, and Heim S (1994). Cytogenetic analysis of multifocal breast carcinomas: detection of karyotypically unrelated clones as well as clonal similarities between tumour foci. Br J Cancer 70:922-927.

Teixeira MR, Pandis N, Bardi G, Andersen JA, Mitelman F, and Heim S (1995). Clonal heterogeneity in breast cancer: Karyotypic comparisons of multiple intra- and extratumorous samples from 3 patients. Int $\mathrm{J}$ Cancer 63:63-68.

Tomita S, Deguchi S, Miyaguni T, Muto Y, Tamamoto T, and Toda $T$ (1999). Analyses of microsatellite instability and the transforming growth factor-beta receptor type II gene mutation in sporadic breast cancer and their correlation with clinicopathological features. Breast Cancer Res Treat 53:3339.

Toyama $\mathrm{T}$, Iwase $\mathrm{H}$, Yamashita $\mathrm{H}$, Iwata $\mathrm{H}$, Yamashita $\mathrm{T}$, Ito K, Hara Y, Suchi M, Kato T, Nakamura T, and Kobayashi S (1996). Microsatellite instability in sporadic human breast cancers. Int J Cancer 68:447-451.

Vogelstein B, Fearon ER, Hamilton SR, Kern SE, Preisinger AC, Leppert M, Nakamura Y, White R, Smits AM, and Bos JL (1988). Genetic alterations during colorectal-tumor development. N Engl J Med 319:525-532.

Walsh T, Chappell SA, Shaw JA, and Walker RA (1998). Microsatellite instability in ductal carcinoma in situ of the breast. J Pathol 185:18-24.

Wolman SR (1986). Cytogenetic heterogeneity: its role in tumor evolution. Cancer Genet Cytogenet 19:129-140.

Wolman SR and Heppner GH (1992). Genetic heterogeneity in breast cancer [Editorial]. J Natl Cancer Inst 84:469-470.

Wooster R, Cleton-Jansen AM, Collins N, Mangion J, Cornelis RS, Cooper CS, Gusterson BA, Ponder BA, von Deimling A, Wiestler OD, Carnelisse CJ, Devilre P, and Stratton MR (1994). Instability of short tandem repeats (microsatellites) in human cancers. Nat Genet 6:152-156.

Yee CJ, Roodi N, Verrier CS, and Parl FF (1994). Microsatellite instability and loss of heterozygosity in breast cancer. Cancer Res 54:1641-1644. 